\title{
Changes of biochemical environment and body weight in healthy periparturient Lipizzan mares
}

\author{
Vladimír Hura ${ }^{1}$, František Novotný ${ }^{1}$, Igor Valocký ${ }^{1}$, Martin Boldižár ${ }^{1}$, Jan Bílek ${ }^{1}$, \\ Petra Horňáková ${ }^{1}$, Zuzana Vilhanová ${ }^{1}$, Slavomír Horňák², Vladimír Petrovič ${ }^{3}$ \\ University of Veterinary Medicine and Pharmacy, ${ }^{1}$ Clinic of Horses, ${ }^{2}$ Clinic of Small Animals, \\ ${ }^{3}$ Department of Pharmacology and Toxicology, Košice, Slovak Republic \\ Received September 20, 2016 \\ Accepted March 3, 2017
}

\begin{abstract}
The aims of this study were to determine the concentrations of calcium, magnesium, phosphorus, chlorine, potassium, sodium, urea, total cholesterol, triacylglycerols, non-esterified fatty acids, total protein, creatinine, total bilirubin, aspartate aminotransferase, insulin, insulin growth factor 1, and glucose in the blood serum and to monitor the body weight changes in nine clinically healthy Lipizzan mares at weekly intervals within 2 weeks before and 2 weeks after the parturition. A significant body weight loss $(P<0.01)$ was found in the mares after the foaling. The peak of insulin growth factor 1 concentration in serum was reached at the day of parturition and similar patterns were revealed for the concentrations of insulin and glucose. After parturition, all these indices were significantly decreased. The concentrations of phosphorus and triacylglycerols were decreased, while the concentrations of potassium and sodium were increased at the day of parturition. Moreover, the concentration of non-esterified fatty acids was increased at the day of parturition $(P<0.05)$ with a tendency to higher values even on the $7^{\text {th }}$ day postpartum. Other studied indexes remained relatively stable throughout the transition period. We can conclude that periparturient mares face some degree of negative energy balance with concomitant significant homeostatic and homeorhetic changes. For this reason, our results can be used as a basis for reference values and a diagnostic tool to examine the health status in horses during the transition period.
\end{abstract}

Horse, metabolic indices, transition phase, homeostatic changes, homeorhetic changes

The peripartal period is a very challenging interval of life of dams due to the decreased feed intake in the late gestation that is accompanied by the increasing metabolic demands of the foetus, and then after the parturition due to the onset of milk production to cover the nutritional requirements of the offspring as well as for the postpartum (p.p.) recovery of the dam. It is well understood mostly from research performed on high-yielding animals, that all these events trigger different cascades of metabolic reactions as a part of the adaptation mechanisms of the organism of the dam concomitant to the transition from the pregnant non-lactating state to the non-pregnant lactating state. From the physiological point of view, the endocrine changes in mares around the parturition have been well described but only limited literature data are available in the field of biochemistry. It is known that especially during the transition period, both pregnant and lactating mares are highly vulnerable to various health disorders which could lead to different productive and reproductive problems (Reed et al. 2010). For this reason, it is recommended to perform a set of metabolic blood tests in dams over the transition to determine the dynamics of homeostatic and homeorhetic changes, and thus predict the course of puerperium in these animals. In general, the assessment of energy balance (non-esterified fatty acids (NEFA), glucose, triacylglycerols (TAG), etc.) is one of the most important tools when considering an animal's health, and the productive and reproductive performance as reviewed by Esposito et al. (2014). According to these authors, the interpretation of the results of 
metabolic profile tests should be taken into account only in light of the health history and total clinical examination of a mare, as well as on the bases of dietary management and welfare in the herd.

Therefore, the objectives of our study were to determine the dynamic changes of calcium, magnesium, phosphorus, chlorine, potassium, sodium, urea, total cholesterol, triacylglycerols, non-esterified fatty acids, total protein, creatinine, total bilirubin, aspartate aminotransferase, insulin, insulin growth factor 1 , glucose and body weight in healthy periparturient Lipizzan mares from the $2^{\text {nd }}$ week before to the $2^{\text {nd }}$ week after foaling.

\section{Materials and Methods}

\section{Animals}

The experiment was performed on a farm that raises only purebred stallions and mares of different breeds. The Lipizzan stallions are used for the natural covering of Lipizzan mares. The health status of mares and stallions is checked regularly and they are subjected to serological testing for infectious equine anaemia and infectious metritis annually, before the onset of the mating season. Moreover, the urogenital apparatus of each mare is regularly examined. A cervical swab is taken and examined microbiologically from each mare intended for mating at six to seven days p.p. and the entire reproductive apparatus of the animal is subjected to rectal and ultrasonographic examination in order to detect the first approaching postpartum oestrus by observation of the following signs: relaxed cervix with mild uterine tonus, oedematous endometrium, dominant ovarian follicle (diameter $\geq 30 \mathrm{~mm}$ ), absence of the uterine content (no cavity). If all these criteria are met, the "healthy" mare is bred by one mount of the desired stallion. When, on the next day, the presence of the ovulation stigma is not confirmed by ultrasonographic examination, the mare is bred again every other day (at 36-h intervals) until the ovulation is confirmed (but only up to three mounts maximum).

The experiment was conducted from March to April to study the transition from pregnancy to lactation. Nine pregnant multiparous Lipizzan mares, parturition of which was expected to occur at the end of March or the beginning of April, from 6 to 12 years of age (mean age $8.6 \pm 2.1$ years) and the mean body weight $543.1 \pm 28.2 \mathrm{~kg}$ were used in the study. The horses were individually housed in boxes on straw and they had free access to water. Every morning, all mares were fed with individually adjusted rations receiving the same diet, but the amount of concentrated feeds given was $1.5 \%$ of the body weight for each mare. The composition of the diet was: $700 \mathrm{~g}$ of hay, $200 \mathrm{~g}$ of oats and $100 \mathrm{~g}$ of granules for horses (approval number SK 100576) per $1 \mathrm{~kg}$ of dry matter (DM). For example, the diet for $500 \mathrm{~kg}$ mare consists of approximately $18.2 \mathrm{~kg}$ of hay, $5.2 \mathrm{~kg}$ of oats, and $2.6 \mathrm{~kg}$ of granules. The granules for horses are based on wheat, barley, corn, sunflower and alfalfa meal, calcium carbonate, sodium chloride, and a mineral premix. Their characteristics per $\mathrm{kg}$ of DM are as follows: moisture $12.25 \%$, crude protein $13.30 \%$, crude oils and fats $3.34 \%$, crude fibre $9.75 \%$, ash $6.15 \%$, lysine $0.51 \%$, methionine $0.21 \%$, calcium $0.93 \%$, phosphorus $0.77 \%$, sodium $0.21 \%$, vitamin A $6250.00 \mathrm{IU}$, vitamin D3 $865.0 \mathrm{IU}$, vitamin E $66.00 \mathrm{mg}$, vitamin C $1.50 \mathrm{mg}$, vitamin B1 $2.10 \mathrm{mg}$, iron $32.0 \mathrm{mg}$, iodine $0.75 \mathrm{mg}$, copper $4.35 \mathrm{mg}$, manganese $29.0 \mathrm{mg}$, zinc $29.0 \mathrm{mg}$, selenium $0.25 \mathrm{mg}$. After the feeding, the horses spent $10 \mathrm{~h}$ on an outdoor pasture.

\section{Procedure}

The experiment was carried out in accordance with established standards for animal care and use. Before the experiment, the mares received a complete clinical examination and were diagnosed as clinically healthy. The parturition occurred spontaneously in all mares, and the mares remained healthy and clinically free of disease for the length of the study period. The mares were weighed by a horse weighbridge at the beginning of the experiment, immediately after the parturition and at the end of the experiment. The blood was sampled at the $14^{\text {th }}$ and $7^{\text {th }}$ day ante partum ( -14 and -7 , respectively), immediately after parturition $(0)$, and at the $7^{\text {th }}$ and $14^{\text {th }}$ day p.p. ( 7 and 14, respectively). The sample collections were done three hours after the morning feeding time. The blood was taken into a gel containing Vacutainer collection tubes and allowed to clot for 20 min at room temperature, and centrifuged at $1,500 \mathrm{~g}$ at $-4^{\circ} \mathrm{C}$ for 5 min to separate the serum.

The serum samples were stored at $-20{ }^{\circ} \mathrm{C}$ till analysed for calcium, magnesium, phosphorus, chloride, potassium, sodium, urea, total cholesterol (TCh), TAG, NEFA, total protein (TP), creatinine (Cre), total bilirubin (TBi), aspartate aminotransferase (AST, EC no: 2.6.1.1), insulin, insulin growth factor 1 (IGF-I), and glucose. The concentrations of calcium, magnesium, potassium, and sodium in the serum were analysed by a flame AAS method (AAnalyst 100, Perkin-Elmer, USA). The concentration of NEFA was assessed using an analytic kit (Randox, UK) by spectrophotometric method (SPECOL 211, Analytik Jena AG, Germany). The concentration of glucose, AST, TCh, TP, Cre, chloride, phosphorus, urea and TAG was determined using commercial diagnostic kits (Randox, UK) on an automatic biochemical analyser (Alizé, Lisabio, France). The concentration of TBi was assessed by a spectrophotometric equipment (SPECORD 210 PLUS, Analytik Jena AG, Germany) using the method according to Jendrassik and Grof (1938). The concentration of insulin was determined by enzyme linked immunosorbent assay using commercial assays (Cusabio, China) according to the manufacturer's instructions. The concentration of IGF-I was determined by radioimmunoassay using human kits S-2143 (Linco Ltd., USA) validated for analysis on animals. 
Evaluations of the obtained results were performed by the assessment of mean values and standard deviations in each monitored time-point. One way analysis of variance with Tuckey's comparison post hoc test was applied for the evaluation of significant differences in the mean values within the same index in relation to the different time-points. Statistical program GraphPad Prism 4.0 software was used for statistical analyses.

\section{Results}

The mean weight of mares in this study was $543.1 \pm 28.2 \mathrm{~kg}$ at -14 and it significantly decreased to $495.6 \pm 28.2 \mathrm{~kg}$ at $0(P<0.01)$, but then only numerical increase of the mean weight to $517.9 \pm 29.1 \mathrm{~kg}$ was found two weeks after the foaling. The concentrations of calcium, magnesium, chloride, urea, TCh, Cre, TP, TBi, and AST remained steady during the entire transition period (Tables 1 and 2). On the other hand, the concentration of NEFA significantly increased at $0(P<0.05)$ and some tendency to maintain this higher level was observed at day 7 (Table 2 ). The peak of plasma IGF-I concentration was reached at 0 (Fig. 1), whereas insulin (Fig. 2) and glucose (Fig. 3) concentrations were relatively stable till the day of parturition. In addition, comparing days 0 to 7 , a significant decrease of all these indices was observed. All these indices remained similarly decreased till day 14 . On the other hand, potassium and sodium concentrations were found at increased levels at day 0 compared to -7 and returned to their periparturient state at days 14 and 7 , respectively

Table 1. Changes of selected macro-mineral indices $(\mathrm{mmol} / \mathrm{l})$ in healthy multiparous Lipizzan mares from 2 weeks before to 2 weeks after delivery.

\begin{tabular}{lccccc}
\hline \multicolumn{5}{c}{ Days related to parturition } \\
\hline Indicator & -14 & -7 & 0 & 7 & 14 \\
\hline Calcium & $3.01 \pm 0.06$ & $3.04 \pm 0.28$ & $3.06 \pm 0.22$ & $2.96 \pm 0.24$ & $2.89 \pm 0.25$ \\
Magnesium & $0.8 \pm 0.06$ & $0.8 \pm 0.07$ & $0.78 \pm 0.1$ & $0.79 \pm 0.11$ & $0.79 \pm 0.14$ \\
Phosphorus & $1.06 \pm 0.16$ & $1.13 \pm 0.15^{\mathrm{a}}$ & $0.86 \pm 0.15^{\mathrm{aB}}$ & $1.23 \pm 0.28^{\mathrm{b}}$ & $1.07 \pm 0.2$ \\
Chloride & $103.6 \pm 3.67$ & $103.4 \pm 3.91$ & $104.1 \pm 2.23$ & $100.9 \pm 4.37$ & $100.7 \pm 5.08$ \\
Potassium & $4.7 \pm 0.2^{\mathrm{AB}}$ & $3.85 \pm 0.19^{\mathrm{ACD}}$ & $4.87 \pm 0.51^{\mathrm{CE}}$ & $4.47 \pm 0.3^{\mathrm{dF}}$ & $3.93 \pm 0.29^{\mathrm{BEF}}$ \\
Sodium & $143.7 \pm 2.08^{\mathrm{ABC}}$ & $135.7 \pm 2.87^{\mathrm{AD}}$ & $144.7 \pm 1.9^{\mathrm{DEF}}$ & $134.2 \pm 3.59^{\mathrm{BE}}$ & $136.1 \pm 1.53^{\mathrm{CF}}$ \\
\hline
\end{tabular}

The results are expressed as mean \pm standard deviation, $\mathrm{n}=9$

Within rows, same superscripts indicate significant differences, ${ }^{\mathrm{xx}} P<0.05,{ }^{\mathrm{x}} P<0.01,{ }^{\mathrm{xx}} P<0.001$.

Table 2. Changes of selected biochemical indices (mmol/l) in healthy multiparous Lipizzan mares from 2 weeks before to 2 weeks after delivery.

\begin{tabular}{lccccc}
\hline \multicolumn{5}{c}{ Days related to parturition } \\
\hline Indicator & -14 & -7 & 0 & 7 & 14 \\
\hline Urea & $5.84 \pm 1.31$ & $6.23 \pm 1.13$ & $6.65 \pm 0.99$ & $5.76 \pm 0.75$ & $5.81 \pm 0.72$ \\
Cre $(\mu \mathrm{mol} / \mathrm{l})$ & $98.2 \pm 11.06^{\mathrm{A}}$ & $106.8 \pm 13.7^{\mathrm{BC}}$ & $100.2 \pm 11.27^{\mathrm{D}}$ & $78.39 \pm 9.39^{\mathrm{aBd}}$ & $89.03 \pm 13.14^{\mathrm{c}}$ \\
TCh & $2.53 \pm 0.44$ & $2.42 \pm 0.48$ & $2.55 \pm 0.42$ & $2.16 \pm 0.31$ & $2.35 \pm 0.37$ \\
TAG & $0.25 \pm 0.09$ & $0.3 \pm 0.08^{\mathrm{Ab}}$ & $0.17 \pm 0.09^{\mathrm{a}}$ & $0.2 \pm 0.07$ & $0.19 \pm 0.06^{\mathrm{b}}$ \\
NEFA & $0.25 \pm 0.15$ & $0.21 \pm 0.15^{\mathrm{a}}$ & $0.38 \pm 0.05^{\mathrm{a}}$ & $0.36 \pm 0.12$ & $0.27 \pm 0.12$ \\
TP $(\mathrm{g} / \mathrm{l})$ & $65.5 \pm 8.09$ & $68.9 \pm 8.28$ & $69.1 \pm 4.47$ & $64.6 \pm 3.28$ & $67.4 \pm 9.04$ \\
TBi $(\mu \mathrm{mol} / \mathrm{l})$ & $16.7 \pm 5.59$ & $18.3 \pm 3.24$ & $20.4 \pm 6.11$ & $13.9 \pm 6.06$ & $16.04 \pm 8.39$ \\
AST $(\mu \mathrm{kat} / \mathrm{l})$ & $5.39 \pm 0.57$ & $5.13 \pm 0.52$ & $5.16 \pm 0.63$ & $5.51 \pm 0.65$ & $5.31 \pm 0.42$ \\
\hline Abbriations: & & &
\end{tabular}

Abbreviations: Cre - creatinine; TCh - total cholesterol; TAG - triacylglycerols; NEFA - non-esterified fatty acids; $\mathrm{TP}$ - total protein; TBi - total bilirubin; AST - aspartate aminotransferase

The results are expressed as mean \pm standard deviation, $\mathrm{n}=9$

Within row, same superscripts indicate significant differences, ${ }^{\mathrm{xx}} P<0.05,{ }^{\mathrm{xx}} P<0.01,{ }^{\mathrm{xx}} P<0.001$. 
(Table 1). In contrast, the concentrations of phosphorus and TAG were significantly decreased at parturition (Table 1 and 2, respectively).

\section{Discussion}

As mentioned previously, the transition from the pregnancy status to the lactation phase is a very challenging episode of the life of a dam, accompanied by many biochemical changes related to the gestation, parturition, and milk production. Many experiments have

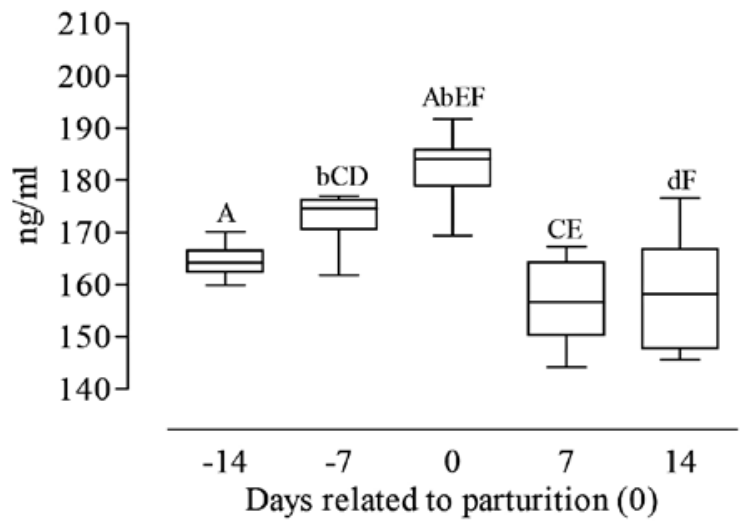

Fig. 1. Changes of the insulin growth factor 1 in healthy multiparous Lipizzan mares from 2 weeks before to 2 weeks after delivery.

The results are expressed as mean \pm standard deviation, $\mathrm{n}=9$

Same letters indicate significant difference, ${ }^{\mathrm{xx}} P<0.05,{ }^{\mathrm{Xx}} P<0.01,{ }^{\mathrm{xx}} P<0.001$.

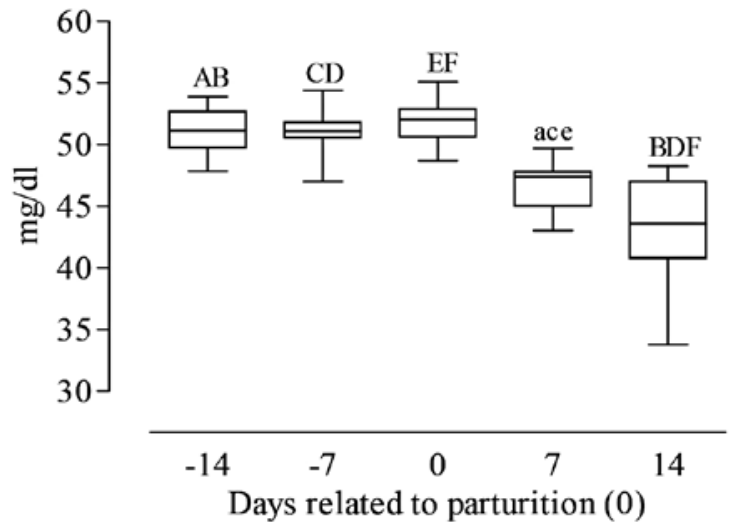

Fig. 2. Changes of insulin in healthy multiparous Lipizzan mares from 2 weeks before to 2 weeks after delivery.

The results are expressed as mean \pm standard deviation, $\mathrm{n}=9$

Same letters indicate significant difference, ${ }^{\mathrm{Xx}} P<0.01,{ }^{\mathrm{Xx}} P<0.001$. 


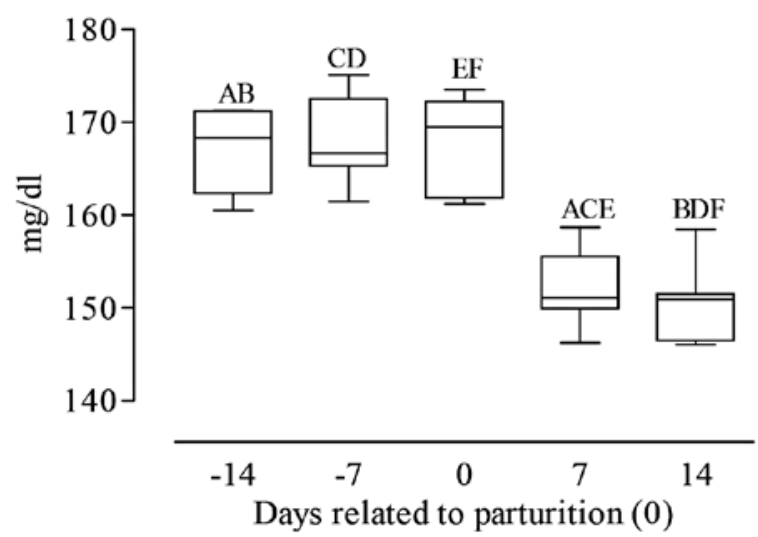

Fig. 3. Changes of glucose in healthy multiparous Lipizzan mares from 2 weeks before to 2 weeks after delivery.

The results are expressed as mean \pm standard deviation, $\mathrm{n}=9$

Same letters indicate significant difference, ${ }^{\mathrm{xx}} P<0.001$.

been conducted on high-yielding cows based on the hypothesis that the health status and body condition p.p. are closely associated to the level of negative energy balance around the parturition, but only few studies which were performed on mares are available in this field of research. Similar studies have been performed by Harvey et al. (2005), Berg et al. (2007) and Mariella et al. (2014), but pregnant mares with a broad age range (4 to 21 years of age) were involved in these experiments. The authors hypothesized that the blood chemistry is not influenced by age in adult horses. In spite of this fact, we decided to study the dynamic changes of different biochemical indices on the group of periparturient mares as homogenous as possible (nine multiparous Lipizzan mares at the mean age of $8.6 \pm 2.1$ year, with known reproductive and health history). Moreover, all mares were clinically healthy throughout our study, and the foaling and expulsion of the placenta occurred spontaneously in these horses. Significant body weight loss was seen in our horses due to the foaling, but thereafter their weight did not change significantly; apparently, the animals gained back some of the weight (around $22 \mathrm{~kg}$ ) two weeks after the parturition. In accordance with these findings, Berg et al. (2007) found a significantly decreased body weight in mares after the foaling.

The focus on macro-elements (such as: calcium, magnesium, phosphorus, chloride, potassium and sodium) is derived from knowledge that they are in the chemical structure of various biologically active molecules which are involved in different homeostatic processes and homeorhetic mechanisms. During the entire experiment, the dynamic changes of calcium, magnesium and chloride concentrations in the blood were not significant at the different time-points, but the concentrations of sodium and potassium were higher and the concentration of phosphorus was lower at day -7 compared to 0 . Moreover, the concentrations of sodium and potassium were significantly decreased at day 14 compared to 0 . This is not in total agreement with the studies published by Martin et al. (1996) and Mariella et al. (2014) who observed a low calcium content in mares at the day of partum, but the concentrations of magnesium and chloride showed a similar pattern as seen in our study. The authors presume that this drop of calcium concentration in the blood is associated with the active delivery with concomitant muscle contractions. On the other hand, in studies performed on horses by Harvey et al. (2005) and A oki and Ishii (2012), no changes of $\mathrm{Ca}$ and $\mathrm{Mg}$ blood concentrations were found. Bazzano et al. (2016) found 
some significant changes of macrominerals in periparturient mares, too. For example, they observed a significant decrease of the concentration of chloride after the delivery probably due to the milk production. It is well understood that considerable amounts of minerals are excreted via equid milk (Salimei and Fantuz 2012). Furthermore, adequate concentrations of nutrients in the diet may prevent some health problems related to macroand micro-element imbalances in horses around the parturition (NRC 2007). This could explain our findings, because our mares were fed with the feed rations recommended for horses in this phase of their productive circle.

In the present study, the creatinine concentration decreased after the parturition, but the urea concentration remained steady. Creatinine is a waste molecule produced from creatine by the muscle metabolism and is readily excreted from the circulation via the kidneys into the urine. For this reason, blood creatinine is used as an indicator of possible malfunction or failure of the kidneys. Furthermore, the blood urea concentration is another useful indicator of renal function. We are inclined to agree with Mariella et al. (2014) that the high concentration of creatinine found prior to parturition should be attributed to its production by the foeto-maternal metabolism. Due to the absence of the foetus, a lower level of creatinine is found after the foaling. Our results are in accordance with the studies performed by Harvey et al. (2005) and Aoki and Ishii (2012). Moreover, these authors presume that the changes in some basal metabolism pathways around parturition could be responsible for the aforementioned findings, rather than the elevated renal clearance. The urea concentration reflects the balance between the catabolic and anabolic processes which are significantly influenced by the nutritional level (Traynor et al. 2006). Neither the urea concentration nor the amount of total proteins revealed any significant changes in our study, although a tendency to lower values was found in both these indices after foaling. It is well established that the feed intake is reduced around parturition, however, the nutritional demands are increased due to the onset of milk production what can result in major periparturient metabolic changes.

We found the peak concentration of NEFA on the day of parturition, while the TAG concentration was significantly decreased at this time-point and the total cholesterol remained stable throughout the experiment. According to Wats on et al. (1993), A oki and Ishii (2012), Mariella et al. (2014), and Bonelli et al. (2016), lower concentrations of TAG and total cholesterol were detected in equids p.p. On the other hand, Harvey et al. (2005) performed the study on light breed mares and found the concentration of TAG remained unaffected during the transition period. The blood NEFA is assessed as an indicator for the mobilization of lipids from the adipose tissues thus reflecting the status of energetic balance in the body. Recent research on dairy cows revealed that reduced concentrations of glucose and insulin in the circulation of dairy cows after the parturition due to the upregulation of some lipolytic signals leading to the elevated hydrolysis of TAG and increased availability of NEFA for the metabolism (Bisinotto et al. 2012), but these processes are not fully explained in horses. Based on these findings, the increase NEFA and decreased TAG at the day of parturition may serve as an evidence that our mares must compensate for the negative energy balance by mobilizing fat reserves.

It is known that a higher bilirubin level in blood is diagnosed due to the liver and bile duct disorders or an increased breakdown of erythrocytes. None of these problems were diagnosed in the mares under our study. In addition, the concentration of total bilirubin was stable during the entire experimental period. In clinical practice, liver transaminases, such as aspartate aminotransferase, are routinely used as a blood biomarker for the liver function in animals and humans. Aspartate aminotransferase is found in both cytoplasm and mitochondria of cells of the liver, heart, skeletal muscle and kidney; thus a structural damage to these tissues can result in an efflux of this enzyme from the intracellular space into the blood stream. From the patho-physiological point of view, only the AST values 
that are at least 10 times above their physiological range have clinical relevance. Yet, Flisinska-Bojanowska et al. (1991), Aoki and Ishii (2012), and Mariella et al. (2014) found major changes in the AST activities in mares in different periods of their reproductive circle. The authors suggest that the activities of these non-specific enzymes are influenced by the anabolic processes which are superior over the catabolic ones in the final trimester as well as by the elevated liver function during the perinatal period. All in all, we should consider the fact that the AST values found in this study did not exceed the physiological range (the reference values of AST in horses, 3.3-6.7 $\mu \mathrm{kat} / \mathrm{l}$; courtesy Clinical Laboratory, University of Veterinary Medicine and Pharmacy in Kosice, unpublished data) thus a severe tissue damage reflecting the pathological conditions is not expected to occur in this case.

In our horses, concentrations of IGF-I in the blood serum decreased significantly after parturition and remained stabilized thereafter. In accordance with these findings, the peak concentration of IGF-I was determined around the parturition, which then declined and remained decreased for more than 2 weeks, as reported by Hess-Dudan et al. (1994) and Berg et al. (2007). In this study, the concentration of insulin and glucose followed the pattern similar to IGF-I. Compared to its postpartum level, a higher value of blood glucose on the day of parturition was found by Aoki and Ishii (2012) and Mariella et al. (2014). Parturition is a very stressful event for the mare which is related to the increase of cortisol concentration, and cortisol in turn triggers gluconeogenesis, increasing the availability of blood sugars for the metabolism as published by Wong et al. (1992) and Davies Morel (1999). It is well understood that the glucose turnover is greatly affected by lactation in horses (Evans 1971) and the lower blood glucose concentration after parturition reflects the mammary gland's demands for this substrate (Hoffman et al. 2003).

Based on these findings we could conclude that the knowledge of endocrine/biochemical events occurring in mares during the transition period is essential to ensure an appropriate management in periparturient horses, which are especially vulnerable to various health problems during this specific phase of their reproductive life. Although the reference values of many biochemical indices are well established, the dynamic changes of some basal metabolites in horses around parturition are not fully understood and these indices can vary widely, even exceeding the physiological range at different time points during stressful events, as was seen in this study. For these reasons, our results can be used as a diagnostic tool to examine the health status in horses during the transition period.

\section{Acknowledgement}

This work was supported by VEGA Scientific Grant No. 1/0366/15 from the Ministry of Education of the Slovak Republic.

\section{References}

Aoki T, Ishii M 2012: Hematological and biochemical profiles in peripartum mares and neonatal foals (Heavy draft horses). J Equine Vet Sci 32: 170-176

Bazzano M, Giudice E, Giannetto C, Fazio F, Scollo C, Piccione G 2016: The peripartum period influenced the serum macromineral profile in mares. Arch Anim Breed 59: 65-70

Berg EL, McNamara DL, Keisler DH 2007: Endocrine profiles of periparturient mares and their foals. J Anim Sci 85:1660-1668

Bisinotto R, Greco L, Ribeiro E, Martinez N, Lima F, Staples C, Thatcher W, Santos J 2012: Influences of nutrition and metabolism on fertility of dairy cows. Anim Reprod Sci 9: 260-272

Bonelli F, Rota A, Corazza M, Serio D, Sgorbini M 2016: Hematological and biochemical findings in pregnant, postfoaling, and lactating jennies. Theriogenology 85: 1233-1238

Davies Morel MCG 1999: Equine reproductive physiology, breeding and stud management. CABI Publishing, 464 p., ISBN 0-85199-372-9

Esposito G, Irons PC, Webb EC, Chapwanya A 2014: Interactions between negative energy balance, metabolic diseases, uterine health and immune response in transition dairy cows. Anim Reprod Sci 144: 60-71 
Evans JW 1971: Effect of fasting, gestation, lactation and exercise on glucose turnover in horses. J Anim Sci 33: 1001-1004

Flisinska-Bojanowska A, Komosa M, Gill J 1991: Influence of pregnancy on diurnal and seasonal changes in glucose level and activity of FDPA, AlAT and AspAT in mares. Comp Biochem Physiol A 98: 31-35

Harvey JW, Pate MG, Kivipelto J, Asquith RL 2005: Clinical biochemistry of pregnant and nursing mares. Vet Clin Pathol 34: 248-254

Hess-Dudan F, Vacher PY, Bruckmaier RM, Weishaupt MA, Burger D, Blum JW 1994: Immunoreactive insulinlike growth factor I and insulin in blood plasma and milk of mares and in blood plasma of foals. Equine Vet $\mathrm{J}$ 26: $134-139$

Hoffman RM, Kronfeld DS, Cooper WL, Harris PA 2003: Glucose clearance in pregnant mares is affected by diet, pregnancy and lactation. J Anim Sci 81: 1764-1771

Jendrassik L, Grof P 1938: Colorimetric Method of Determination of Bilirubin. Biochem Z 297: 81-82

Mariella J, Pirrone A, Gentilini F, Castagnetti C 2014: Hematological and biochemical profiles in Standardbred mares during peripartum. Theriogenology 81: 526-534

Martin KL, Hoffman RM, Kronfeld DS, Ley WB, Warnick LD 1996: Calcium decreases and parathyroid hormone increases in serum of periparturient mares. J Anim Sci 74: 834-839

NRC - Nutrition Research Council 2007: Minerals. Nutrient requirements of horses, $6^{\text {th }}$ revised edition, Washington, DC, USA, The National Academy Press, Animal Nutrition Series, pp. 69-108

Reed SM, Bayly WM, Sellon DC 2010: Equine internal medicine. $3^{\text {rd }}$ edn. by Saunders, St. Louis, MO, 1488 p.

Salimei E, Fantuz F 2012: Equid milk for human consumption. Int Dairy J 24: 130-142

Traynor J, Mactier R, Geddes CC, Fox JG 2006: How to measure renal function in clinical practice. BMJ 333: 733-737

Watson TD, Burns L, Packard CJ, Shepherd J 1993: Effects of pregnancy and lactation on plasma lipid and lipoprotein concentrations, lipoprotein composition and post-heparin lipase activities in Shetland pony mares. J Reprod Fertil 97: 563-568

Wong CW, Smith SE, Thong YH, Opdebeeck JP, Thornton JR 1992: Effects of exercise stress on various immune functions in horses. Am J Vet Res 53: 1414-1417 